\title{
Jahresinhaltsverzeichnis 2021
}

\section{AUFSÄTZE}

G ü n z e l, Martin: Die Wegbereiter. Tony Blair, Großbritannien und die Entscheidung für den Irakkrieg 2001 bis 2003 . . . . . . . . . . . . . . . 445

K a $1 \mathrm{~m} \mathrm{~b}$ a c h, Peter Lutz: Fliegende Standgerichte. Entstehung und Wirkung eines Instruments der nationalsozialistischen Militärjustiz

K u t z n e r , Maximilian: „Zeitung für Deutschland“?

Die Frankfurter Allgemeine Zeitung und die deutsche Frage 1969 bis 1990

$\mathrm{N}$ a t t e r m a n n, Ruth: Emanzipatorischer Aufbruch und antisemitische Verfolgung. Jüdinnen in der italienischen Frauenbewegung 1914 bis 1945 . . 25

P a t e 1, Kiran Klaus: Kooperation und Konkurrenz. Die Entstehung der europäischen Wissenschafts- und Forschungspolitik seit 1945 . . . . . . . 183

P e n t e r, Tanja/T y t a r e n k o, Dmytro: Der Holodomor, die NS-Propaganda in der Ukraine und ihr schwieriges Erbe

R a d u , Sorin: Der Aufbau des Sozialismus. Kaderschulen und Parteibürokratie in Rumänien - die regionale Parteischule in Timișoara 1948 bis 1973 . . 55

R a p h a e 1, Lutz: Deutsche Arbeitswelten zwischen globalen Problemlagen und nationalen Handlungsbezügen. Zeitgeschichtliche Perspektiven . . . . . . 1

R e i t m a y e r, Morten: Populismus als Untersuchungsfeld der Zeitgeschichte. Ein kritischer Forschungsbericht . . . . . . . . . . . . 573

S c h w a r t z, Michael: Homosexuelle im modernen Deutschland. Eine Langzeitperspektive auf historische Transformationen . . . . . . . . . . . 377

S t e g e r, Sascha: Kurt Daluege, die Stennes-Revolten 1930/31 und der Aufstieg der SS

S z ö 11 ö s i - J a n z e , Margit: Archäologie des Wettbewerbs. Konkurrenz in und zwischen Universitäten in (West-)Deutschland seit den 1980er Jahren

\section{DISKUSSION}

G o t t o , Bernhard: „Bürohengste“, „Amtsfräulein“, Bürger_? Geschlechterperspektiven auf Verwaltung in der bundesdeutschen Nachkriegsdemokratie 733 
H e i n e m a n n , Isabel: Die patriarchale Familie als „Keimzelle“ der Demokratie - oder deren größte Bedrohung? Konflikte um den Wert

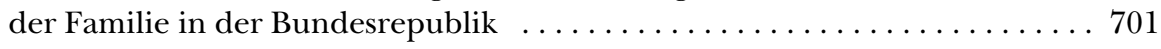

H e i n e m a n n, Isabel/S t e b e r, Martina: Geschlecht und Demokratie. Deutungskämpfe um die Ordnung der Gesellschaft in der Bundesrepublik Deutschland

H e i n s o h n, Kirsten: Gruppenbild ohne Dame. Demokratie in der

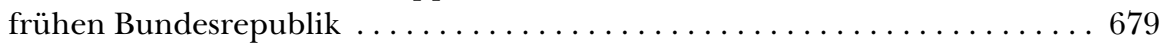

P a u 1 u s , Julia: Geschlechter-Räume und Demokratie . . . . . . . . . . . 723

S t e b e r , Martina: Staatsbürgerinnen für die Demokratie. Konservative Frauen und die junge Bundesrepublik $\ldots \ldots \ldots \ldots \ldots \ldots \ldots \ldots \ldots \ldots$

v a n R a h d e n , Till: Im Herbst der Patriarchen. Demokratie und Männlichkeit in der frühen Bundesrepublik . . . . . . . . . . . . . . 689

\section{DOKUMENTATIONEN}

G a h n , Philipp: Widersprüche eines Modus Vivendi. Dokumente zum Besuch Michael Kardinal von Faulhabers bei Adolf Hitler auf dem

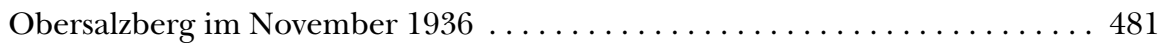

S c h 1 o t t , René: Ablehnung und Anerkennung. Raul Hilberg und das Institut für Zeitgeschichte

\section{GUTACHTEN}

Ein Sozialdemokrat im Auswärtigen Amt. Gutachten des Instituts für Zeitgeschichte zur Bedeutung Hermann Müllers für die Außenpolitik der Weimarer Republik . . . . . . . . . . . . . . . . . . . . . 12

\section{MISZELLE}

G e o r g i e v , Anna: „Im fremden Erdteil ein Kleinod sein, ein Segen werden“. Über den Verbleib der Kultgegenstände (zwangs)aufgelöster jüdischer Gemeinden 


\section{PODIUM ZEITGESCHICHTE}

Mehr als eine Fußnote! Die Sowjetische Besatzungszone und die DDR in der deutschen und europäischen Geschichte . . . . . . . . . . . . . 279

$\mathrm{G}$ ü n t h e r, Frieder: Die verwaltete Teilung. Behördengeschichte in deutsch-deutscher Perspektive

J o h n , Jürgen: Besatzungsgeschichte vor der doppelten Staatsgründung.

Stand, Probleme, Defizite und Aufgaben der SBZ-Forschung . . . . . . . . . . . 284

P e t e r s , Florian: Am Schnittpunkt von Ost und West. Ostmitteleuropäische Perspektiven für eine transnationale DDR-Geschichte ...

S c h e r s t j a n o i, Elke: Werden und Wachsen. Zur Frühgeschichte der DDR als aktuellem Forschungsfeld . . . . . . . . . . . . . . . 295

S c h w a r t z, Michael: Transformationsgesellschaft. DDR-Geschichte

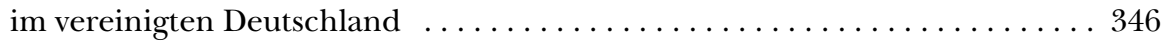

W e b e r , Petra: Die Gesellschaft der DDR im Widerstreit. Offene Fragen

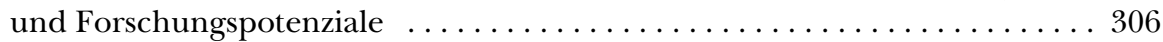

\section{NOTIZEN}

Idyll und Verbrechen. Die neue Dauerausstellung der Dokumentation

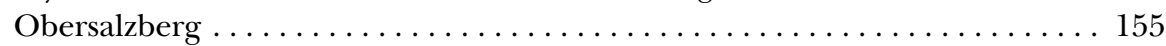

15. Aldersbacher Schreib-Praxis. Ein anwendungsorientiertes Seminar des Instituts für Zeitgeschichte und des Verlags De Gruyter Oldenbourg (26. bis 30. Juli 2021)

Bemerkung zur Dokumentation von René Schlott, Ablehnung und Anerkennung. Raul Hilberg und das Institut für Zeitgeschichte, im Januar-Heft der VfZ

\section{VfZ-ONLINE}

Kommentar im VfZ-Forum und Aufzeichnung des „Podiums Zeitgeschichte“ 2020 . . . . . . . . . . . . . . . . . . . . . . . . . 169 
Ergänzende Materialien zu zwei VfZ-Beiträgen der Januar-Ausgabe 361

Neu bei den Zusatzangeboten: Nachtrag zu René Schlotts Dokumentation im Januar-Heft 559

Neu: Innovatives Video-Format, Aufzeichnung des „Podiums Zeitgeschichte" 2021 und ergänzende Materialien zu Philipp Gahns Dokumentation in der Juli-Ausgabe

\section{AUS DER REDAKTION}

Hans Maier zum 90. Geburtstag 549 